\title{
ON THE ZEROS OF GENERALIZED AXIALLY SYMMETRIC POTENTIALS*
}

\author{
PETER A. MCCOY
}

\begin{abstract}
Generalized axially symmetric potentials may be expanded as Fourier-Jacobi series in terms of the complete system $r^{k} C_{k}^{n / 2-1}(\cos \theta)$ on axisymmetric regions $\Omega \subset E^{n}(n \geqslant 3)$ about the origin. The values of these potentials are characterized by the nonnegativity of sequences of determinants drawn from the Fourier coefficients in a manner analogous to the characterization of the values of analytic functions of one complex variable by the theorems of Carathéodory-Toeplitz and Schur.
\end{abstract}

1. Introduction. The singularities of generalized axially symmetric potentials (GASP) $\psi$ are characterized from the coefficients of their Fourier-Jacobi series expansions by S. Bergman [1], [2] and R. P. Gilbert [3], [4] who found a definitive characterization by employing function theoretic means to develop a theory mirroring the classical work of Hadamard, Mandelbrojt and Fabry on the singularities of analytic functions $f$ of one complex variable. Cauchy's classical estimate [5, p. 123] on the zeros of a polynomial, along with the theorems of Carathéodory-Toeplitz [12, p. 157] and Schur [12, p. 159] bound the zeros of $f$ relative to convex sets from its coefficients; the possibility arises of developing a parallel theory for the values of $\psi$.

An extension of Cauchy's estimate applying to the zeros of harmonic polynomials in $E^{n}$ was found by Morris Marden [8]. We shall illustrate how the reasoning in [8] which is based on methods found in Professor Marden's early work [6], [7] can be used to locate the zeros of GASP. Moreover, it is apparent that this reasoning plays a role in bounding the zeros of $\psi$ analogous to the role played by the reasoning of Hadamard in the multiplication of singularities theorem upon which Gilbert's "envelope method" [1], [2] is based. Remark: Investigations [9]-[11] subsequent to [8] employ the $A_{n}$ operator [4, p. 168] to geometrically establish equality of known sets of excluded values of the $A_{n}$ associates and corresponding sets of excluded values of GASP.

2. On the zeros. In axisymmetric regions $\Omega \subset E^{n}[8]$ about the origin, generalized axially symmetric potentials $\psi_{n}$ may be developed as a series $[4, \mathrm{p}$. 168]

Received by the editors September 28, 1975 and, in revised form, December 22, 1975.

AMS (MOS) subject classifications (1970). Primary 35B05, 35C10; Secondary 30A08.

Key words and phrases. Bergman and Gilbert's integral operators, Gilbert-Hadamard theorem, Carathéodory-Toeplitz and Schur theorems, zeros of potentials.

*This paper is dedicated to Professor Morris Marden on the occasion of his retirement from the University of Wisconsin-Milwaukee.

Copyright $\odot 1977$, American Mathematical Society 


$$
\psi_{n}(r, \theta)=\psi_{n}(\mathbf{X})=\sum_{k=0}^{\infty} B(n-2, k+1) a_{k} r^{k} C_{k}^{n-1 / 2}(\cos \theta)
$$

in terms of the polar coordinates $(r, \theta)$ which depend on the cylindrical coordinates $x=x_{1}, \rho=\left(x_{2}^{2}+\cdots+x_{n}^{2}\right)^{1 / 2}$ by $x=r \cos \theta, \rho=r \sin \theta$. Because of symmetry, $\psi_{n}$ assumes constant values of hypercircles $\mathbf{X}=(x, \rho)$ in $\Omega$.

For each $\psi_{n}$ with domain $\Omega$, there is a unique associated function $f$,

$$
f(\xi)=\sum_{k=0}^{\infty} a_{k} \xi^{k}
$$

analytic on the corresponding axiconvex domain $\omega \subset \mathbf{C}[8]$ whose $A_{n}$ transform [4] is defined by

$$
\psi_{n}(\mathbf{X})=\alpha_{n} \int_{L} f(\sigma) d \nu_{n}(\zeta)
$$

$\sigma=x+\rho / 2\left(\zeta+\zeta^{-1}\right), \alpha_{n}=\Gamma(n-2) /(4 i)^{n-2} \Gamma(n / 2-1)$ with nonnegative measure

$$
d \nu_{n}(\zeta)=\left(\zeta-\zeta^{-1}\right)^{n-3} d \zeta / \zeta
$$

for $\zeta$ on the contour $L \equiv\left\{\zeta=e^{i t} \mid 0 \leqslant t \leqslant \pi\right\}$. (The Fourier-Legendre expansion for $n=3$ follows from the Bergman-Whittaker operator.) From the development of $\psi_{n}$ in (1) we defined the matrix

$$
M_{k}=\left(\begin{array}{c}
b_{0}, a_{1}, \ldots, a_{k} \\
0, b_{0}, \ldots, a_{k-1} \\
\ldots \ldots \ldots . . . \\
0,0,0, \ldots, b_{0}
\end{array}\right)
$$

its conjugate transpose $\bar{M}_{k}^{\prime}\left(b_{0} \equiv a_{0}-\alpha, \alpha \in \mathbf{C}\right)$ and the determinants

$$
\sigma_{k}(\alpha)=\sigma_{k}\left(a_{0}, \ldots, a_{k}, \alpha\right)=\operatorname{det}\left(M_{k}+\bar{M}_{k}^{\prime}\right)
$$

and

$$
\gamma_{k}(\alpha)=\gamma_{k}\left(a_{0}, \ldots, a_{k}, \alpha\right)=\operatorname{det}\left(\begin{array}{cc}
M_{k} & I_{k} \\
I_{k} & \bar{M}_{k}^{\prime}
\end{array}\right)
$$

where $I_{k}$ is the $(k+1)$ by $(k+1)$ identity matrix. Initially the values of $\psi_{n}$ relative to the hypersphere $S_{n}$ with unit radius about the origin are considered in

THEOREM 1. Let the generalized axially symmetric potential $\psi_{n}$ be expanded as in (1). Then $\psi_{n}$ is regular in the hypersphere $S_{n}$ and on each hypercircle $\mathbf{X}$ in $S_{n}$,

$$
\psi_{n}(\mathbf{X}) \neq \alpha+\eta, \quad \forall \operatorname{Re}(\eta)<0,\left(a_{0}-\alpha \text { real }\right)
$$

or

$$
\psi_{n}(\mathbf{X}) \neq \eta, \quad \forall|\eta-\alpha|>1
$$

for $\eta$ complex according to whether 


$$
\sigma_{0}(\alpha)>0, \sigma_{1}(\alpha)>0, \ldots, \sigma_{k}(\alpha)>0, \ldots
$$

or

$$
\gamma_{0}(\alpha)>0, \gamma_{1}(\alpha)>0, \ldots, \gamma_{k}(\alpha)>0, \ldots
$$

In particular, if $\alpha>0$ and (8a) or $|\eta|>1$ and (9a), then $\psi_{n}$ has no zeros in $S_{n}$.

Proof. When (8a) or (9a) are valid, the associate $f$ given by (2) is regular in the disk $D=|\xi|<1$ so that its $A_{n}$ transform represents $\psi_{n}$ in $S_{n}$. When (8a) is satisfied, the Carathéodory-Toeplitz theorem guarantees that $\operatorname{Re}[f(\sigma)-\alpha] \geqslant$ 0 when $|\sigma|<1$ which is the case for all $x^{2}+\rho^{2}<1$ and $\zeta \in L$. Moreover, for $\zeta \in L$, the measure $d v_{n}(\zeta)$ is nonnegative. Consequently, the integral $\psi_{n}-\alpha=A_{n}(f-\alpha)$, viewed as the uniform limit of a sum of vectors, each terminating in the right half plane $P$, is in $P$ (see [6], [7]). This establishes (8).

In (9a), Schur's theorem guarantees the bound $|f(\sigma)-\alpha| \leqslant 1, x^{2}+\rho^{2}$ $<1, \zeta \in L$. The measure is nonnegative and the operator is normalized according to $A_{n}(1)=1$. Hence $\left|\psi_{n}(\mathbf{X})-\alpha\right| \leqslant A_{n}(1)$. Remark: Due to Fatou's theorem [12, p. 146], $\alpha>0$ is a sufficient condition for the existence of the radial limits of $\psi_{n}$ relative to $S_{n}$, with the possible exception of a set of singular circles whose intersection with a meridian plane has one dimensional measure zero.

THEOREM 2. If for some constant $\alpha$, the sequence

$$
\gamma_{0}(\alpha)>0, \ldots, \gamma_{k}(\alpha)>0, \quad \gamma_{k+1}(\alpha)=\cdots=0,
$$

then $\psi_{n}$ is a linear combination of Newtonian potentials whose mass is distributed over $k+1$ singular circles exterior to $S_{n}$ such that $\psi_{n}$ has no zeros in the double cone

$$
0<\rho< \pm(R-x) \tan (\pi / 4(k+1)) .
$$

The distance $R$ from the origin to the most remote (finite) singular circle is determined by the Gilbert-Hadamard theorem. Moreover, if $\psi_{n}$ vanishes on the sphere centre at $x=x_{0}$ and radius $\rho=\rho_{0}$ with no singular circles in the concentric sphere of radius $\rho_{0} \csc (\pi / 2(k+1))$ about $S_{n}$, the associate vanishes in the disk $\left|\xi-x_{0}\right| \leqslant \rho_{0} \csc (\pi / 2(k+1))$.

Proof. Because of (10), the associate $f$ is a rational function with $(k+1)$ distinct zeros and $(k+1)$ poles which are symmetric in $|\xi|=1$ [12, p. 159]. Application of the $A_{n}$ operator to the partial fraction expansion of $f$ establishes the first part. To verify that $\psi_{n}$ has no zeros in the cones (11), we reason as in [8] bearing in mind the location of the zeros and poles of the associate. To establish the converse relation on the effect of the zeros of $\psi$ on those of $f$, apply Marden's mean value theorem [5, p. 11] in a manner similar to its use in [9].

Relating the values of $\psi_{n}$ on axisymmetric sets to the values assumed in convex sets suggests a lengthy statement on the Fourier coefficients. For brevity, we consider the version in

THEOREM 3. The generalized axially symmetric potential $\psi_{n}$ as in (1) has the axisymmetric set $\Omega \subset E^{n}$ for its domain where the analytic function 


$$
g(\xi)=\sum_{k=0}^{\infty} g_{k} \xi^{k} / k !
$$

maps the unit disk onto the corresponding axiconvex set $\omega \subset \mathbf{C}$. The constants $\beta_{k}$ are defined by

$$
\begin{aligned}
& \beta_{k}=\sum_{j=0}^{k} a_{j} j ! / k ! \sum P_{k}^{(j)} g_{1}^{m_{1}} \ldots g_{k}^{m_{1}}, \\
& P_{k}^{(j)}=k ! /(1 !)^{m_{1}} \ldots(k !)^{m_{k}}\left(m_{k} !\right) .
\end{aligned}
$$

The summations are over $m_{1}+2 m_{2}+\cdots+k m_{k}=k$, and $m_{1}+\cdots+m_{k}$ $=j$. If either sequence,

$$
\sigma_{0}\left(\beta_{0}, \alpha\right)>0, \ldots, \sigma_{k}\left(\beta_{0}, \ldots, \beta_{k}, \alpha\right)>0, \ldots
$$

or

$$
\gamma_{0}\left(\beta_{0}, \alpha\right)>0, \ldots, \gamma_{k}\left(\beta_{0}, \ldots, \beta_{k}, \alpha\right)>0, \ldots,
$$

then on each hypercircle $\mathbf{X} \in \Omega, \psi_{n}$ satisfies the corresponding inequality (8) or (9).

Proof. We write $\tilde{f}=f \circ g$. Then (15) or (16) imply that $f$ maps $D$ onto the respective convex sets $D$ or $\operatorname{Re}(\xi) \geqslant 0$. Consequently, $f: \omega \rightarrow D$ or $\operatorname{Re}(\xi) \geqslant 0$ so that reasoning as in Theorem 1 completes the proof.

3. A generalization to $\Delta_{3} \Phi+F\left(r^{2}\right)=0$. The purpose of this closing remark is to indicate a broader range of application of [6], [7] by referring to work of S. Bergman [2] who expands axially symmetric solutions of

$$
L \Phi \equiv \frac{\partial^{2} \Phi}{\partial x^{2}}+\frac{\partial^{2} \Phi}{\partial \rho^{2}}+\frac{1}{\rho} \frac{\partial \Phi}{\partial \rho}+F\left(r^{2}\right) \Phi=0
$$

( $F$ is entire) in series of generalized Bessel functions $J_{n}^{*}$ [2, p. 435].

THEOREM 4. If

$$
\Phi(\mathbf{X})=\sum_{k=0}^{\infty} \frac{\Gamma(1 / 2) A_{k}}{\Gamma(k+3 / 2)} \frac{J_{k}^{*}(r)}{J_{k}^{*}(1)} r^{k} P_{k}(\cos \theta)
$$

is the development of a solution of $L \Phi \equiv 0$ regular in the unit sphere $S_{3}$ and there is a constant $|\alpha|>1$ for which

$$
\gamma_{0}\left(A_{0}, \alpha\right)>0, \gamma_{1}\left(A_{0}, A_{1}, \alpha\right)>0, \ldots, \gamma_{k}\left(A_{0}, \ldots, A_{k}, \alpha\right)>0
$$

then on each circle $\mathbf{X} \in S_{3}$,

$$
\Phi(\mathbf{X}) \neq 0
$$

Proof. The proof relies on the operators $\Phi=D_{0}(H)[2$, p. 435] and $H=B_{3}(f)$. Because of (19) and (essentially) Theorem $1,|H(\mathbf{X})-\alpha|<1$, $|\mathbf{X}|<1$. Therefore, since the generating function of $D_{0}$ is real, we may conclude from [7] that $|\Phi(\mathbf{X})-\alpha|<1$.

REMARK. More general cases can be treated using the Method of Ascent (see Gilbert [3]). 


\section{REFERENCES}

1. S. Bergman, Integral operators in the theory of linear partial differential equations, Ergebnisse der Mathematik und ihrer Grenzgebiete, N. F., Heft 23, Springer-Verlag, Berlin and New York, 1961. MR 25 \#5277.

2. Classes of solutions of linear partial differential equations in three variables, Duke Math. J. 13 (1946), 419-458. MR 8, 274.

3. R. P. Gilbert, Constructive methods for elliptic equations, Lecture Notes in Math., vol. 365, Springer-Verlag, Berlin and New York, 1974.

4. __ Function theoretic methods in partial differential equations, Math. in Science and Engineering, Vol. 54, Academic Press, New York, 1969. MR 39 \#127.

5. M. Marden, Geometry of polynomials, 2nd ed., Math. Surveys, No. 3, Amer. Math. Soc., Providence, R. I., 1966. MR 37 \# 1562.

6. , A generalization of Weierstrass' and Fekete's mean-value theorems, Bull. Amer. Math. Soc. 38 (1932), $434-441$.

7. __ , Further mean-value theorems, Bull. Amer. Math. Soc. 39 (1933), 750-754.

8. , Value distribution of harmonic polynomials in several real variables, Trans. Amer. Math. Soc. 159 (1971), 137-154. MR 43 \#5046.

9. P. A. McCoy, Value distribution of axisymmetric potentials, Amer. J. Math. 95 (1973), 419-428. MR 48 \#6450.

10. , Value distribution of linear combinations of axisymmetric harmonic polynomials and their derivatives, Pacific J. Math. 48 (1973), 441-450.

11. _ Generalized axisymmetric potentials, J. Approximation Theory 15 (1975), 256266.

12. M. Tsuji, Potential theory in modern function theory, Maruzen, Tokyo, 1958. MR 22 \#5712.

Department of Mathematics, U. S. Naval Academy, Annapolis, Maryland 21401 